\title{
DESEMPENHO DE ESCOLARES COM DISTÚRBIO DE APRENDIZAGEM E DISLEXIA EM TESTES DE PROCESSAMENTO AUDITIVO
}

\author{
Performance of students with learning disabilities \\ and dyslexia on auditory processing tests
}

\author{
Adriana Marques de Oliveira ${ }^{(1)}$, Ana Cláudia Vieira Cardoso ${ }^{(2)}$, Simone Aparecida Capellini ${ }^{(3)}$
}

\begin{abstract}
RESUMO
Objetivo: caracterizar e comparar, por meio de testes comportamentais, o processamento auditivo de escolares com diagnóstico interdisciplinar de (I) distúrbio da aprendizagem, (II) dislexia e (III) escolares com bom desempenho acadêmico. Método: participaram deste estudo 30 escolares na faixa etária de 8 a 16 anos de idade, de ambos os gêneros, de $2^{\underline{a}}$ a $4^{\underline{a}}$ séries do ensino fundamental, divididos em três grupos: Gl composto por 10 escolares com diagnóstico interdisciplinar de distúrbio de aprendizagem, Gll: composto por 10 escolares com diagnóstico interdisciplinar de dislexia e GIII composto por 10 escolares sem dificuldades de aprendizagem, pareados segundo gênero e faixa etária com os grupos GI e Gll. Foram realizadas avaliação audiológica e de processamento auditivo. Resultados: os escolares de GIII apresentaram desempenho superior nos testes de processamento auditivo em relação aos escolares de GI e GII. GI apresentou desempenho inferior nas habilidades auditivas avaliadas para testes dicóticos de dígitos e dissílabos alternados, logoaudiometria pediátrica, localização sonora, memória verbal e não-verbal, ao passo que Gll apresentou as mesmas alterações de Gl, com exceção do teste de logoaudiometria pediátrica. Conclusão: os escolares com transtornos de aprendizagem apresentaram desempenho inferior nos testes de processamento auditivo, sendo que os escolares com distúrbio de aprendizagem apresentaram maior número de habilidades auditivas alteradas, em comparação com os escolares com dislexia, por terem apresentado atenção sustentada reduzida. O grupo de escolares com dislexia apresentou alterações decorrentes da dificuldade relacionada à codificação e decodificação de estímulos sonoros.
\end{abstract}

DESCRITORES: Percepção Auditiva; Transtornos da Audição; Transtornos de Aprendizagem; Dislexia

(1) Fonoaudióloga; Mestranda do Programa de Pós-Graduação em Educação da Faculdade de Filosofia e Ciências da Universidade Estadual Paulista - FFC/UNESP, Marília, SP; Bolsista do Conselho Nacional de Desenvolvimento Cientifico e Tecnológico - CNPq .

(2) Fonoaudióloga; Docente do Departamento de Fonoaudiologia da Faculdade de Filosofia e Ciências da Universidade Estadual Paulista FFC/ UNESP - Marília - SP; Doutora em Distúrbios da Comunicação Humana Universidade Federal de São Paulo UNIFESP, São Paulo, SP.

(3) Fonoaudióloga; Docente do Departamento de Fonoaudiologia e Programa de Pós-Graduação em Educação da Faculdade de Filosofia e Ciências da Universidade Estadual Paulista - FFC/UNESP, Marília, SP; Doutora e PósDoutora em Ciências Médicas pela Faculdade de Ciências Médicas da Universidade Estadual de Campinas, FCM/ UNICAMP, Campinas, SP.

Conflito de interesses: inexistente

\section{INTRODUÇÃO}

Processamento auditivo é a habilidade de ouvir, compreender e responder às informações que ouvimos por meio dos canais auditivos. Isso inclui a detecção do som pela orelha externa e a transmissão do som por meio das vias auditivas até o cérebro ${ }^{1,2}$, ou seja, o processamento auditivo referese à eficiência e à eficácia com a qual o sistema nervoso central utiliza as informações auditivas ${ }^{3}$.

Deste modo, o processamento auditivo está diretamente relacionado com as funções de discriminação, memória e percepção auditiva, que são importantes na expressão e compreensão da palavra falada, na leitura e na escrita ${ }^{4-6}$. 
A desordem do processamento auditivo é um déficit no processamento neural do estímulo auditivo, que abrange desde a detecção da presença de som até a análise da informação linguística, envolvendo funções perceptuais, cognitivas e linguísticas. A manifestação mais comum da desordem do processamento auditivo é a dificuldade de compreensão da fala em situação de ruído. Escolares com a desordem do processamento auditivo podem ter dificuldades na ortografia e leitura, o que se torna mais evidente em situações desafiadoras de escuta, podendo apresentar um impacto negativo sobre a aquisição da linguagem e desempenho acadêmico, ou seja, a desordem do processamento auditivo geralmente está associada aos transtornos de aprendizagem ${ }^{1,5,7-10}$

Os escolares que apresentam problemas de aprendizagem geralmente apresentam desempenho inferior em testes de processamento auditivo em função do atraso na maturação das habilidades auditivas ${ }^{11,12}$, o que foi demonstrado por estudo norteamericano ${ }^{13}$, em que a prevalência de desordem do processamento auditivo em escolares com transtornos de aprendizagem foi estimada em 30 a $50 \%$ dessa população.

A dislexia é um transtorno específico no aprendizado da leitura, de origem neurológica, cuja característica principal é rendimento escolar inferior ao esperado, tendo em conta a idade cronológica, o potencial do indivíduo e sua escolaridade ${ }^{14}$. 0 distúrbio de aprendizagem é um diagnóstico diferencial tanto em relação à dislexia como à dificuldade de aprendizagem, sendo uma expressão genérica que se refere a um grupo de alterações que manifestam dificuldades significativas na aquisição e no uso de: audição, fala, leitura, escrita, raciocínio ou habilidades matemáticas ${ }^{15,16}$.

Os escolares com dislexia apresentam alteração na discriminação dos sons e, por isso, a fusão rápida de estímulos encontra-se comprometida, resultando na dificuldade para organizar a sequência dos sons na formação das palavras ${ }^{17}$. A dificuldade central da dislexia está relacionada ao processamento de sons, conhecidos como processamento fonológico, ou seja, dificuldade de processar pistas auditivas breves e rápidas, implicando a falta de habilidade em perceber elementos críticos da fala de forma acurada, não permitindo, assim, o acesso à formação da codificação fonológica ${ }^{18,19}$. Os escolares com o diagnóstico de distúrbio de aprendizagem apresentam características alteradas em habilidades como a identificação ou decodificação da palavra, compreensão de leitura, cálculo, raciocínio matemático, soletração e expressão escrita, e ainda podem ter acometidas áreas acadêmicas que envolvem, de maneira mais ampla, a expressão oral e a compreensão auditiva ${ }^{20,21}$.

Com base no exposto, este estudo teve por objetivo caracterizar e comparar, por meio de testes comportamentais, o processamento auditivo de escolares com diagnóstico interdisciplinar de distúrbio de aprendizagem e dislexia e escolares com bom desempenho acadêmico.

\section{MÉTODO}

Participaram deste estudo clínico, com corte transversal, 30 escolares na faixa etária de 8 a 16 anos de idade, de ambos os gêneros, de $2^{\underline{a}}$ a $4^{\mathrm{a}}$ série do ensino fundamental da cidade de Marília $\mathrm{SP}$, divididos em três grupos:

- Grupo I (GI): formado por 10 escolares, sendo 7 (70\%) do gênero masculino e $3(30 \%)$ do gênero feminino, com faixa etária de 8 a 12 anos, com diagnóstico interdisciplinar de distúrbio de aprendizagem, em início de processo terapêutico do Centro de Estudos da Educação e Saúde da Faculdade de Filosofia e Ciências da Universidade Estadual Paulista - FFC/UNESP.

- Grupo II (GII): formado por 10 escolares, sendo 8 $(80 \%)$ do gênero masculino e $2(20 \%)$ do gênero feminino, com faixa etária de 8 a 16 anos, com diagnóstico interdisciplinar de dislexia, em início de processo terapêutico do Centro de Estudos da Educação e Saúde da Faculdade de Filosofia e Ciências da Universidade Estadual Paulista - FFC/UNESP.

- Grupo III (GIII): formado por 10 escolares, sendo $6(60 \%)$ do gênero masculino e $4(40 \%)$ do gênero feminino, com faixa etária de 8 a 10 anos, com bom desempenho acadêmico, de escola pública municipal da cidade de Marília, sendo pareados segundo a escolaridade com GI e GIl. Os professores indicaram os escolares que apresentaram conceito suficiente em pelo menos 2 bimestres consecutivos em provas de Língua Portuguesa e Matemática e que não apresentavam alterações auditiva, visual, motora e cognitiva descritas em prontuário escolar.

O diagnóstico de distúrbio de aprendizagem e dislexia foi realizado por equipe interdisciplinar do Centro de Estudos da Educação e Saúde - CEES/ UNESP - de Marília e do Ambulatório de Neurologia Infantil - Desvios da Aprendizagem da Faculdade de Medicina - FM/UNESP - de Botucatu, incluindo avaliação fonoaudiológica, neurológica e neuropsicológica.

Neste estudo foram realizados os seguintes procedimentos:

a) Termo de consentimento pós-informado: Conforme resolução do Conselho Nacional 
de Saúde CNS 196/96, anterior ao início das avaliações, os pais ou responsáveis dos pacientes selecionados assinaram o termo de consentimento pós- informado para a autorização da realização do estudo.

b) Avaliação audiológica: A avaliação audiológica básica e a avaliação comportamental do processamento auditivo foram realizadas em cabina acústica. Para a audiometria tonal limiar e logoaudiometrica, foi utilizado o audiômetro GSI 61 (padrão ANSI 3.6-1989 e S3.431992) com fone TDH - 50. Para a normalidade audiológica periférica, foi considerado o critério proposto para crianças, considerando até $15 \mathrm{~dB}$ para normalidade ${ }^{22}$. Para a imitanciometria, foi utilizado o imitanciômetro GSI-38, sendo que, para realizarem a avaliação do processamento auditivo, os escolares precisavam apresentar curva timpanométrica do tipo $A^{23}$.

c) Avaliação do Processamento Auditivo: Foi realizada a triagem do processamento auditivo constituída pelos seguintes testes dióticos: Localização Sonora em cinco direções (acima, direita, esquerda, frente e atrás da cabeça), e Memória Sequencial para Sons verbais e não-verbais ${ }^{24}$.

O Teste de Localização Sonora em cinco direções foi realizado utilizando como estímulo o instrumento musical guizo. Foi considerada resposta positiva quando houve a localização de pelo menos quatro das cinco posições testadas.

O Teste de Memória Sequencial para sons não-verbais foi aplicado utilizando-se quatro instrumentos musicais: campânula vertical com cinco guizos, sino, coco e agogô. Foi apresentada a sequência de quatro sons variando a ordem. Foi considerado um bom desempenho a identificação correta de pelo menos duas sequências de estímulos entre as três apresentadas.

O Teste de Memória Sequencial verbal foi feito com as sílabas "pa", "ta", "ca" e "fa", dispostas em ordens diferentes. Foi considerado bom desempenho a repetição correta de pelo menos duas das três ordens sequenciais pelos escolares.

A avaliação do processamento auditivo foi realizada por meio de um audiômetro de dois canais GSI-61, ao qual foi acoplado um tocador de CD (CD- Player) da marca Sony. Foi utilizado um CD com os seguintes testes: Logoaudiometria Pediátrica, Dicótico de Dígitos e Dicótico de Dissílabos alternados (SSW).

O Teste Dicótico de Dígitos foi realizado numa intensidade com 50 dBNS. Foi apresentada uma lista com 20 pares de dígitos. Este teste, na tarefa de integração binaural, tem como objetivo avaliar a habilidade para agrupar componentes do sinal acústico em figura-fundo e identificá-los. A tarefa de separação binaural possibilita avaliar a escuta direcionada para cada orelha separadamente.

O Teste Dicótico de Dissílabos Alternado (SSW) em português foi realizado numa intensidade de $50 \mathrm{dBNS}$ e contém 40 itens. Ao escolar foi pedido para repetir o que ouviu obedecendo à ordem de apresentação das palavras.

O teste de Logoaudiometria Pediátrica - PSI (Pediatric Speech Intelligibility Test) foi realizado numa intensidade de $40 \mathrm{dBNS}$. Foi aplicado o teste de reconhecimento de sentenças na presença de mensagem competitiva ipsilateral nas seguintes relações: 0 dBNA, -10 dBNA e - 15 dBNA. Foi posicionado o escolar de frente para a prancha contendo todas as figuras representantes das frases que foram ouvidas.

O procedimento foi realizado no Centro de Estudos da Educação e da Saúde - CEES/Unesp Marília. Foram realizadas duas sessões para a avaliação, com duração de 50 minutos cada.

Este estudo foi realizado depois da aprovação do Comitê de Ética em Pesquisa da Faculdade de Filosofia e Ciências da Universidade Estadual Paulista - CEP/FFC/UNESP/ Marília-SP sob o protocolo no 660/2009.

Para a realização da análise estatística, foi utilizado o programa SPSS (Statistical Package for Social Sciences) em sua versão 13.0, com nível de significância de 5\% (0,050). Foram aplicados - Teste de Qui-quadrado, ajustado pela Estatística de Fisher, e o Teste de Kruskal-Wallis com o intuito de verificar possíveis diferenças entre os três grupos, quando comparados concomitantemente, e o Teste de Mann-Whitney, para identificar quai $s$ grupos diferenciam-se entre si, quando comparados. A significância estatística foi marcada por asterisco $\left(^{*}\right)$.

\section{RESULTADOS}

Nas Tabelas de 1 a 6 encontram-se os resultados da comparação concomitante entre os três grupos. A análise estatística foi realizada por meio do Teste de Qui-quadrado, ajustado pela estatística de Fisher.

A tabela 1 apresenta a classificação dos grupos GI, Gll e GIII para o Teste Dicótico de Dígitos, separado por orelha. Observa-se que houve diferença estatisticamente significante entre os três grupos, sendo que o grupo GIII apresentou melhor habilidade tanto para agrupar o sinal acústico em figura-fundo, como para identificá-lo verbalmente. O grupo Gll apresentou desempenho superior que GI nessas habilidades. 
Tabela 1 - Classificação dos grupos GI, GIl e GIII para o teste dicótico de dígitos, separado por orelha

\begin{tabular}{|c|c|c|c|c|c|c|c|}
\hline \multirow{2}{*}{ GRUPO } & \multicolumn{2}{|c|}{ DIC DIG Classif OD } & \multirow{2}{*}{ Total } & \multirow{2}{*}{ GRUPO } & \multicolumn{2}{|c|}{ DIC DIG Classif OE } & \multirow{2}{*}{ Total } \\
\hline & Normal & Alterado & & & Normal & Alterado & \\
\hline \multirow[t]{2}{*}{1} & 0 & 10 & 10 & 1 & 0 & 10 & 10 \\
\hline & 0,00 & 100,00 & 100,00 & & 0,00 & 100,00 & 100,00 \\
\hline \multirow[t]{2}{*}{ II } & 5 & 5 & 10 & II & 2 & 8 & 10 \\
\hline & 50,00 & 50,00 & 100,00 & & 20,00 & 80,00 & 100,00 \\
\hline \multirow[t]{2}{*}{ III } & 10 & 0 & 10 & III & 10 & 0 & 10 \\
\hline & 100,00 & 0,00 & 100,00 & & 100,00 & 0,00 & 100,00 \\
\hline \multirow[t]{2}{*}{ Total } & 15 & 15 & 30 & Total & 12 & 18 & 30 \\
\hline & 50,00 & 50,00 & 100,00 & & 40,00 & 60,00 & 100,00 \\
\hline
\end{tabular}

Legenda: DIC DIG classif: Classificação dicótico de dígitos; OE: orelha esquerda; OD: orelha direita

A Tabela 2 mostra a classificação dos grupos GI, GIl e GIII para o Teste Dicótico de Dissílabos Alternados. Pode-se observar que $100 \%$ dos escolares de Gl apresentaram alteração, a qual variou do grau severo ao moderado. $70 \%$ dos escolares de GIl apresentaram alteração variando de grau leve a severo. Já nenhum escolar de GIII apresentou qualquer alteração. A análise estatística demonstrou diferença estatisticamente significante entre os três grupos, refletindo as dificuldades em figura-fundo, atenção auditiva e organização apresentadas pelos escolares de GI e Gll.

A Tabela 3 apresenta classificação dos grupos GI, GII e GIII para o Teste de Memória Sequencial Não-Verbal (MSNV) e Verbal (MSV). A Tabela 4 apresenta a classificação do Teste de Localização Sonora. Verifica-se que não houve diferença estatisticamente significante entre os três grupos.

A Tabela 5 apresenta a classificação dos grupos para o Teste de Logoaudiometria Pediátrica (PSI) separado por orelhas. Verifica-se que houve diferença estatisticamente significante em ambas as orelhas, evidenciando que GI difere dos outros dois grupos por apresentar maior média de escolares com alteração em ambas as orelhas. Este resultado sugere que os escolares desse grupo apresentam alteração nas habilidades auditivas de figura-fundo e atenção seletiva. Com isso, pode-se afirmar que os escolares de Gl apresentam alteração para a compreensão da mensagem com competição de ruído.

A Tabela 6 apresenta a distribuição gráfica da média, desvio padrão, mediana e valor de p para os testes Dicótico de Dígitos e Dissílabos Alternados (SSW).

Foi realizada a aplicação do Teste de KruskalWallis, com o intuito de verificar possíveis diferenças entre os três grupos, quando comparados concomitantemente. Foi encontrada diferença estatisticamente significante, evidenciando que GI apresentou média de desempenho inferior em relação às médias do Gll e GIII nos testes de Dicótico de Dígitos e Dicótico de Dissílabos Alternados (SSW), em ambas as orelhas.

Tabela 2 - Classificação dos grupos GI, GIl e GIII para o teste dicótico de dissílabos alternados (SSW)

\begin{tabular}{|c|c|c|c|c|c|}
\hline \multirow{2}{*}{ GRUPO } & \multicolumn{4}{|c|}{ SSW_Classif } & \multirow{2}{*}{ Total } \\
\hline & Normal & Leve & Moderado & Severo & \\
\hline \multirow[t]{2}{*}{1} & 0 & 0 & 2 & 8 & 10 \\
\hline & 0,00 & 0,00 & 20,00 & 80,00 & 100,00 \\
\hline \multirow[t]{2}{*}{ II } & 1 & 2 & 5 & 2 & 10 \\
\hline & 10,00 & 20,00 & 50,00 & 20,00 & 100,00 \\
\hline \multirow[t]{2}{*}{ III } & 10 & 0 & 0 & 0 & 10 \\
\hline & 100,00 & 0,00 & 0,00 & 0,00 & 100,00 \\
\hline \multirow[t]{2}{*}{ Total } & 11 & 2 & 7 & 10 & 30 \\
\hline & 36,67 & 6,67 & 23,33 & 33,33 & 100,00 \\
\hline
\end{tabular}

Legenda: SSW_Classif: Classificação dicótico de dígitos alternados (SSW) 
Tabela 3 - Classificação dos grupos GI, GIl e GIII para o teste de memória sequencial não verbal (MSNV) e memória sequencial verbal (MSV)

\begin{tabular}{|c|c|c|c|c|c|c|c|}
\hline \multirow{2}{*}{ GRUPO } & \multicolumn{2}{|c|}{ MSNV } & \multirow{2}{*}{ Total } & \multirow{2}{*}{ GRUPO } & \multicolumn{2}{|c|}{ MSV } & \multirow{2}{*}{ Total } \\
\hline & Normal & Alterado & & & Normal & Alterado & \\
\hline \multirow[t]{2}{*}{ I } & 8 & 2 & 10 & I & 6 & 4 & 10 \\
\hline & 80,00 & 20,00 & 100,00 & & 60,00 & 40,00 & 100,00 \\
\hline \multirow[t]{2}{*}{ II } & 9 & 1 & 10 & II & 8 & 2 & 10 \\
\hline & 90,00 & 10,00 & 100,00 & & 80,00 & 20,00 & 100,00 \\
\hline \multirow[t]{2}{*}{ III } & 10 & 0 & 10 & III & 10 & 0 & 10 \\
\hline & 100,00 & 0,00 & 100,00 & & 100,00 & 0,00 & 100,00 \\
\hline \multirow[t]{2}{*}{ Total } & 27 & 3 & 30 & Total & 24 & 6 & 30 \\
\hline & 90,00 & 10,00 & 100,00 & & 80,00 & 20,00 & 100,00 \\
\hline
\end{tabular}

Legenda: MSNV: memória sequencial não verbal; MSV: memória sequencial verbal; OD: orelha direita; OE: orelha esquerda

Tabela 4 - Classificação dos grupos GI, GIl e GIII para o teste de localização sonora

\begin{tabular}{cccc}
\hline \multirow{2}{*}{ GRUPO } & LS & & Total \\
\cline { 2 - 4 } & Normal & Alterado & 10 \\
& 9 & 1 & 100,00 \\
\hline I & 90,00 & 10,00 & 10 \\
& 8 & 2 & 100,00 \\
\hline III & 80,00 & 20,00 & 10 \\
& 10 & 0 & 100,00 \\
\hline Total & 100,00 & 3 & 30 \\
& 27 & 10,00 & 100,00 \\
\hline
\end{tabular}

Legenda: LS: localização sonora; OD: orelha direita; OE: orelha esquerda

Tabela 5 - Classificação dos grupos GI, GIl e GIII para o teste de logoaudiometria pediátrica (PSI) ispilateral, separado por orelha

\begin{tabular}{|c|c|c|c|c|c|c|c|}
\hline \multirow{2}{*}{ GRUPO } & \multicolumn{2}{|c|}{ PSI_OD } & \multirow{2}{*}{ Total } & \multirow{2}{*}{ GRUPO } & \multicolumn{2}{|c|}{ PSI_OE } & \multirow{2}{*}{ Total } \\
\hline & Normal & Alterado & & & Normal & Alterado & \\
\hline \multirow[t]{2}{*}{ I } & 6 & 4 & 10 & 1 & 6 & 4 & 10 \\
\hline & 60,00 & 40,00 & 100,00 & & 60,00 & 40,00 & 100,00 \\
\hline \multirow[t]{2}{*}{ II } & 10 & 0 & 10 & II & 10 & 0 & 10 \\
\hline & 90,00 & 10,00 & 100,00 & & 100,00 & 0,00 & 100,00 \\
\hline \multirow[t]{2}{*}{ III } & 10 & 0 & 10 & III & 10 & 0 & 10 \\
\hline & 100,00 & 0,00 & 100,00 & & 100,00 & 0,00 & 100,00 \\
\hline \multirow[t]{2}{*}{ Total } & 26 & 4 & 30 & Total & 26 & 4 & 30 \\
\hline & 86,67 & 13,33 & 100,00 & & 86,67 & 13,33 & 100,00 \\
\hline
\end{tabular}


Tabela 6 - Distribuição gráfica da média, desvio padrão, mediana e valor de p nos testes de dicótico de digitos, dicótico de dígitos alternados (SSW).

\begin{tabular}{ccccccc}
\hline Variável & Grupo & $\mathbf{n}$ & Média & $\begin{array}{c}\text { Desvio } \\
\text { padrão }\end{array}$ & Mediana & $\begin{array}{c}\text { Significância } \\
(\mathbf{p})\end{array}$ \\
\hline DIC_DIG_IB_OD & I & 10 & 77,13 & 7,02 & 77,50 & $<0,001^{*}$ \\
& II & 10 & 88,25 & 9,78 & 91,25 & \\
& III & 10 & 96,63 & 2,21 & 96,88 & \\
& Total & 30 & 87,33 & 10,61 & 91,25 & \\
\hline DIC_DIG_IB_OE & I & 10 & 73,88 & 16,30 & 76,25 & $<0,001^{*}$ \\
& II & 10 & 83,50 & 12,87 & 88,75 & \\
& III & 10 & 95,88 & 1,32 & 96,25 & \\
& Total & 30 & 84,42 & 14,77 & 90,00 & \\
SSW_OD_C & I & 10 & 51,00 & 10,62 & 50,00 & $<0,001^{*}$ \\
& II & 10 & 79,75 & 10,89 & 80,00 & \\
& III & 10 & 96,75 & 2,37 & 97,50 & \\
& Total & 30 & 75,83 & 21,03 & 80,00 & \\
\hline SSW_OE_C & I & 10 & 48,75 & 19,41 & 45,00 & $<0,001^{*}$ \\
& II & 10 & 72,00 & 20,58 & 76,25 & \\
& III & 10 & 94,50 & 2,84 & 95,00 & \\
& Total & 30 & 71,75 & 24,73 & 78,75 & \\
\hline
\end{tabular}

Legenda: DIC_DIG: Dicótico de dígitos; IB: integração binaural; SSW: Dicótico de dígitos alternados; C: competitiva; OD: orelha direita; OE: orelha esquerda

A Tabela 7 apresenta a classificação dos grupos GI,Gll e Glll para os testes Dicótico de Dígitos e Dicótico de Dissílabos Alternados (SSW), em ambas as orelhas. Como na tabela 6 , para essas variáveis, foram encontradas diferenças estatisticamente significantes. Foi aplicado o Teste de Mann-Whitney para identificar quais grupos diferenciam-se entre si quando comparados par a par. Observou-se que houve diferença estatisticamente significante para o teste Dicótico de Dissílabos Alternados e Dicótico de Dígitos. A exceção foi encontrada na comparação entre os grupos GI e Gll e Gll e GIII, para o Teste Dicótico de Dígitos, na orelha esquerda e direita respectivamente.

Tabela 7 - Classificação dos grupos GI, GIl e GIII para os testes de dicótico de digitos e dicótico de díssilabos alternados (SSW)

\begin{tabular}{cccc}
\hline \multirow{2}{*}{ Variável } & \multicolumn{3}{c}{ Par de Grupos } \\
\cline { 2 - 4 } & $\mathbf{I} \mathbf{x}$ |I & $\mathbf{I} \mathbf{x}$ III & II x III \\
\hline DIC_DIG_IB_OD & $0,023^{*}$ & $0,000^{*}$ & 0,063 \\
DIC_DIG_IB_OE & 0,120 & $0,000^{*}$ & $0,004^{*}$ \\
SSW_OD_C & $0,000^{*}$ & $0,000^{*}$ & $0,000^{*}$ \\
SSW_OE_C & $0,012^{*}$ & $0,000^{*}$ & $0,002^{*}$ \\
\hline
\end{tabular}

Legenda: DIC_DIG: Dicótico de dígitos; IB: integração binaural; SSW: Dicótico de dissílabos alternados; C: competitiva; OD: orelha direita; OE: orelha esquerda

\section{DISCUSSÃO}

Os resultados deste estudo revelaram que, nos escolares de GI e Gll, as habilidades auditivas encontram-se alteradas, quando comparados aos escolares do GIII, o que vai de encontro à literatura da área, a qual afirma que escolares com prejuízo em linguagem, ortografia, leitura e compreensão de leitura apresentam problemas de processamento auditivo, ou, em outras palavras, que as habilidades 
de processamento auditivo estão mais prejudicadas nos escolares com transtornos de aprendizagem tais como distúrbio de aprendizagem (GI) e dislexia (GII), quando comparadas aos escolares sem estes transtornos $1,7,9,10,17,20,21,25-28$.

A percepção da origem da fonte sonora tem grande importância devido à estreita relação entre audição, fala, leitura e escrita. No caso de as habilidades de identificação e localização não se desenvolverem adequadamente, o desenvolvimento das demais habilidades pode ser prejudicado ${ }^{29}$. Neste estudo, pôde-se observar que, nos escolares com distúrbio de aprendizagem e dislexia, a habilidade de localização sonora encontra-se alterada.

A ineficácia da integração de informações em escolares com distúrbio de aprendizagem e dislexia pôde ser verificada nos testes Dicótico de Dígitos e Dissílabos Alternados. A média alcançada pelos escolares com distúrbio de aprendizagem e dislexia do desenvolvimento foi menor que a dos escolares com bom desempenho acadêmico, assemelhandose a outros estudos descritos na literatura 7,17,26,27,30.

Todavia, apesar de os grupos I e II apresentarem desempenhos inferiores nos testes do processamento auditivo, o desempenho de Gl foi inferior em comparação a GII, demonstrando que os escolares com distúrbio de aprendizagem apresentam dificuldades na execução de tarefas que envolvem a atenção seletiva.

O processamento auditivo abrange desde a detecção de som até a análise da informação linguística, envolvendo funções perceptuais, cognitivas e linguísticas ${ }^{9,10}$. Nos escolares com distúrbio de aprendizagem, os processamentos cognitivo, linguístico, auditivo e visual estão comprometidos e, em decorrência dessas falhas, o acionamento de mecanismos cognitivos para analisar, sintetizar, manipular, estocar e evocar informações linguísticas encontra-se alterado ${ }^{15}$. Tais habilidades são importantes para a leitura e a escrita, pois o conteúdo lido pelo escolar deve ser mantido na memória de curto prazo para que o sentido ${ }^{12}$ seja alcançado. Os resultados alterados encontrados em Gl e Gll ocorrem devido a déficits na percepção dos fonemas da fala, integração auditiva e memória de curto prazo ${ }^{25,31}$. É provável, nestes casos, que ao sistema fonológico não funcione de forma eficiente em decorrência da baixa qualidade das representações de longa duração ${ }^{32}$. Uma das causas pode ser a dificuldade na percepção da fala no nível dos fonemas, que pode interferir no processo de manipulação das informações ${ }^{33-35}$.
O desempenho inferior dos escolares de Gl e Gll nos testes de Memória Verbal e Não-Verbal pode ter ocorrido devido a características dos próprios quadros de distúrbio de aprendizagem ou dislexia, em que a memória de trabalho comprometida pode impedir o bom desempenho em leitura em nível de decodificação, compreensão da leitura e o desenvolvimento do vocabulário ${ }^{36-38}$.

A partir dos resultados deste estudo, pôde-se verificar que os escolares com transtornos de aprendizagem tiveram um desempenho inferior nos testes de processamento auditivo, em comparação aos escolares com bom desempenho escolar. Os escolares com distúrbio de aprendizagem apresentaram maior número de habilidades auditivas alteradas, o que pode ser explicado pelo fato de terem atenção sustentada reduzida, enquanto os escolares com dislexia apresentaram somente alterações decorrentes da dificuldade relacionada com a codificação e decodificação de estímulos sonoros.

\section{CONCLUSÃO}

Os achados deste estudo permitem concluir que: - Os escolares de GIII, com bom desempenho acadêmico, apresentaram desempenho superior nos testes de processamento auditivo em relação aos escolares de Gl e Gll.

- Os escolares de GI, com distúrbio de aprendizagem, apresentaram desempenho inferior nas habilidades auditivas avaliadas nos testes Dicótico de Dígitos, Dissílabos Alternados, Loogoaudiometria Pediátrica, Localização sonora, Memória Verbal e Não-Verbal. Os escolares de GII, com dislexia, também apresentaram alterações nestes testes, com exceção do teste de logoaudiometria pediátrica, porém em menor grau que os escolares de Gl, evidenciando a necessidade da continuidade de estudos com maior número de casos para melhor diferenciação do perfil auditivo destes escolares.

Desta forma, este estudo evidenciou que o fator atencional alterado nos escolares com distúrbio de aprendizagem é evidente nos testes logoaudiometria pediátrica e dicótico de dissílabos alternados, onde o desempenho desse grupo variou de moderado a severo, enquanto os escolares com dislexia variaram de leve a severo o que parece ser uma evidência do diferencial entre o distúrbio de aprendizagem e a dislexia. 


\begin{abstract}
Purpose: to characterize and compare, by means of behavioral tests, the auditory processing of students with an interdisciplinary diagnosis of (I) learning disorder, (II) dyslexia and (III) students with good academic performance. Method: thirty students ranging from the ages 8 to 16 year old, from both genders, took part in this study and were divided as follows: Group I (GI): composed of 10 students with interdisciplinary diagnosis of learning disabilities. Group II (GII): composed of 10 students with dyslexia diagnosis. Group III (GIII): composed of 10 students, attending grades two to four, with good academic performance, paired up according to gender and age with groups I and II. A basic auditory evaluation and an evaluation of central auditory processing were conducted. Results: the students of GIII showed statistically superior performance in auditory processing tests compared to the students of GI and GII. GI showed lower performance in auditory abilities assessed in the tests of dichotic digits, alternating dissyllable, pediatric speech intelligibility, sound localization, memory for verbal and non-verbal sounds, and GIl also showed the same changes as GI, except in the pediatric speech intelligibility test. Conclusion: the students with learning disabilities showed lower performance in auditory processing tests, and the students with learning disorders had the highest number of altered abilities when compared with the students with dyslexia, which can be explained by the fact that this group shows reduced sustained attention. The dyslexia group showed alterations derived from the difficulty related to the auditory stimulus encoding and decoding.
\end{abstract}

KEYWORDS: Auditory Perception; Hearing Disorders; Learning Disorders; Dyslexia

\section{REFERÊNCIAS}

1. Yalçinkaya F, Muluk NB, Sahin S. Effects of listening ability on speaking, writing and reading skills of children who were suspected of auditory processing difficulty. Int J Pediatr Otorhinolaryngol. 2009; 73(8): 1137-42.

2. Ríos AA, Rezende AG, Pela SM, Ortiz KZ, Pereira LD. Teste de padrão harmônico em escuta dicótica com dígitos - TDDH. Rev Soc Bras Fonoaudiol. 2007;12(4):304-9.

3. American Speech-Language-Hearing Association (ASHA). (Central) Auditory Processing Disorders [Technical Report]. 2005. [acesso em 15 dez 2009] Available from www.asha.org/policy.

4. Kujala T, Lovio R, Lepistö $T$, Laasonen $M$, Näätänen R. Evaluation of multi-attribute auditory discrimination in dyslexia with the mismatch negativity. Clin Neurophysiol. 2006;117(4):885-93.

5. Dawes P, Bishop DV, Sirimanna T, Bamiou DE. Profile and aetiology of children diagnosed with auditory processing disorder (APD). Int $\mathrm{J}$ Pediatr Otorhinolaryngol. 2008;72(4):483-9.

6. Zalcman TE, Schochat E. A eficácia do treinamento auditivo formal em indivíduos com transtorno de processamento auditivo. Rev Soc Bras Fonoaudiol. 2007;12(4):310-4.

7. Engelmann L, Ferreira MIDC. Avaliação do processamento auditivo em crianças com dificuldades de aprendizagem. Rev Soc Bras Fonoaudiol. 2009; 14 (1): 69-74.

8. Iliadou V, Bamiou DE, Kaprinis S, Kandylis D; Vlaikidis N,Apalla $\mathrm{K}$, et al. Auditory processing disorder and brain pathology in a preterm child with learning disabilities. J Am Acad Audiol.2008; 19(7):557-63.

9. Moore DR, Rosenberg JF, Coleman JS. Discrimination training of phonemic contrasts enhances phonological processing in mainstream school children. Brain Lang. 2005; 94(1):72-85.

10. Moore DR. Auditory processing disorder (ADP) - Potential contribution of mouse research. Brain res. 2006.1091(1):200-6.

11. Simon LF, Rossi AG. Triagem do processamento auditivo em escolares de 8 a 10 anos. Psicol Esc Educ. 2006;10(2):293-304.

12. Furbeta TDC, de Felippe AC. Avaliação simplificada do processamento auditivo e dificuldades de leitura-escrita. Pró-Fono: Rev Atual Cient. 2005;17(1):11-8.

13. Iliadou V, Bamiou DE, Kaprinis S, Kandylis D, Kaprinis G. Auditory processing disorders in children suspected of learning disabilities - a need for screening. Int J Pediatr Otorhinolaryngol. 2009; 73(7)1029-34.

14. American Psychiatric Association. Diagnostic and statistical manual of mental disorders: DSM-IV. Washington, DC: American Psychiatric Association; 2000. 
15. Silva C. Eficácia de um programa de remediação fonológica e leitura em escolares com distúrbio de aprendizagem [dissertação]. Marília (SP): Faculdade de Filosofia e Ciências, Universidade Estadual Paulista; 2009.

16. Capellini SA. Distúrbios de aprendizagem versus dislexia. In: Ferreira LP, Lopes DMB, Limongi SCO, editores. Tratado de fonoaudiologia. São Paulo: Roca; 2004. p. 862-76.

17. Germano GD, Capellini SA. Eficácia do programa de remediação auditivo-visual computadorizado em escolares com dislexia. Pró-Fono: Rev Atual Cient. 2008;20(4):237-42.

18. Magnan A, Ecalle J. Audio-training in children with reading disabilities. Comp Educ. 2006; 46 (4):407-25.

19. Temple E, Deutsch GK, Poldrack RA, Miller $S$, Tallal P, Merzenich MM, et al. Neural deficits in children with dyslexia ameliorated by behavioral remediation: evidence from functional MRI. Proc Natl. Acad. Sci USA. 2003; 100(5):2860-5.

20. Silver CH, Ruff RM, Iverson GL, Barth JT, Broshek DK, Bush SS, et al. Nan Policy and Planning Commitee. Learning disabilities: The need for neuropsycological evaluation. Arch of Clin Neuropsychol. 2008;23(2):217-9.

21. Wu TK, Huan SC, Meng YR. Evaluation of ANN and SVM classifiers as predictors to the diagnosis of students with learning disabilities. Exp Syst Apppl. 2008. 34(3):846-56.

22. Northern JL, Downs MP. Audição em crianças. 3. ed. São Paulo: Manole; 1989.

23. Jerger J. Clinical experience with impedance audiometry. Arch Otolaryng. 1970. 92:311-24.

24. Pereira LD, Schochat E. Processamento auditivo central: manual de avaliação. São Paulo: Lovise; 1997.

25. Dlouha O, Novak A, Vokral J. Central auditory disorder (CAPD) in children with specific languagem impairment (SLI) central auditory tests. Int J Pediatr Otorhinolaryngol. 2007; 71(6):903-7.

26. Pinheiro FH, Capellini SA. Desenvolvimento das habilidades auditivas de escolares com distúrbio de aprendizagem, antes e após treinamento auditivo, e suas implicações educacionais. Rev Psicopedagogia.2009; 26(80):231-41.

doi: 10.1590/S1516-18462010005000126

RECEBIDO EM: 04/02/10

ACEITO EM: 15/05/2010

Endereço para Correspondência:

Adriana Marques de Oliveira

Rua: Mato Grosso no 285

CEP: 17509-090

E-mail: adrimaroli@yahoo.com.br
27. GarciaVL, Pereira, LD, FukudaY. Atençãoseletiva: PSI em crianças com distúrbio de aprendizagem. Rev Bras Otorrinolaringol.2007;73(3):404-11.

28. Sauer L, Pereira, LD, Ciasca, SM, Pesturn M, Guerreiro,MM. Processamento auditivo e spect em crianças com dislexia. Arq Neuropsiquiatr. 2006,64(1):108-11.

29. Santos CMF, Bragato GR, Martins PMF, Dias AB. Triagem auditiva em escolares de 5 a 10 anos. Rev. CEFAC [periódico na internet]. Epub 31-jul-2009 [acesso em 2010 jan 18]: 10p. Disponivel: http:// www.scielo.br/pdf/rcefac/2009nahead/160-08.pdf

30. Pinheiro FH. Eficácia do programa de treinamento auditivo em escolares com distúrbio de aprendizagem [dissertação]. Marília (SP): Faculdade de Filosofia e Ciências, Universidade Estadual Paulista; 2009.

31. Germano GD, Pinheiro FH, Cardoso ACV, Santos LCA, Padula NAMR, Capellini SA. Relação entre os achados em neuroimagem, habilidades auditivas e metafonológicas em escolares com dislexia do desenvolvimento. Rev Soc Bras Fonoaudiol.2009;14(3):315-22.

32. Thomson JM, Richardson U, Goswami U. Phonological similarity neighborhoods and children's short-term memory: typical development and dyslexia. Memory Cogn.2005;33(7):1210-9.

33. Pekkola J, Laasonen M, Ojanen V, Autti T, Jaaskelainen IP, Kujala $T$, et al. Perception of matching and conflicting audiovisual speech in dyslexic and fluent readers: an fMRI study at 3T. Neuroimage.2006;29(3):797-807.

34. Temple E, Poldrack RA, Protopapas S, Nagarajan S, Salz T, Tallal P, et al. Disruption of neural response to rapad acoustic stimuli in dislexia: evidence from functional MRI. Proc Natl Acad Sci USA.2000;97(25):13907-12.

35. Boets $B$, Wouters $J$, VanWieringen $A$, Ghesquière P. Auditory processing, speech perception and phonological ability in pre-school children at high-risk for dyslexia: a longitudinal study of the auditory temporal processing theory. Neuropsychol.2007;45(8):1608-20.

36. Tzenova J, Kaplan BJ, Petryshen TL, Field LL. Confirmation of a dyslexia susceptibility locus on chromosome 1p34-p36 in a set of 100 Canadian families. Am J Med Genet.2004;27(1):117-24.

37. Banai K, Ahissar M. Auditory processing déficits in dyslexia: task or stimulu related? Cerebral Cortex. 2006;16(12):1718-28.

38. Leonard C, Eckert M, Given B, Virginia B, Eden $G$. Individual differences in anatomy predict reading and oral language impairments in children. Brain. 2006;129(12):3329-42. 